\title{
ASYMPTOTIC STABILITY WITH RATE CONDITIONS FOR DYNAMICAL SYSTEMS
}

\author{
BY NEIL FENICHEL
}

Communicated by Shlomo Sternberg, August 2, 1973

We announce existence and smoothness theorems for invariant manifolds characterized by asymptotic stability at specified rates. Our theorems require roughly half of a hyperbolic structure, so various known results about stable and unstable manifolds are included as special cases. Even under hyperbolicity assumptions, however, our results give new information about metric properties, invariant foliations, and asymptotic stability with asymptotic phase. Proofs will appear in [2].

1. Invariant sets with expanding structure. Let $U$ and $V$ be open subsets of a $C^{r}$ manifold $M, 1 \leqq r \leqq \infty$, and let $F: U \rightarrow V$ be a $C^{r}$ diffeomorphism. $\Lambda \subset U$ is called weakly overflowing invariant (under $F$ ) if $\Lambda \subset F(\Lambda)$. Let $T F: T U \rightarrow T V$ be the map induced by $F$ on tangent spaces. A subbundle $E \subset T M \mid \Lambda$ is called weakly overflowing invariant if $E \subset T F(E)$. We assume that all bundles have constant dimension.

Suppose that $\Lambda$ is compact, and that $\Lambda$ and $E$ are weakly overflowing invariant. Choose any vector bundle $N \subset T M \mid \Lambda$, complementary to $E$, and let $\pi^{E}$ and $\pi^{N}$ be the projections corresponding to the splitting $T M \mid \Lambda=E \oplus N$. For any $m \in \Lambda$, and any $v^{0} \in E_{m}, w^{0} \in N_{m}$, let

$$
v^{-k}=D F^{-k}(m) v^{0}, \quad w^{-k}=\pi^{N} D F^{-k}(m) w^{0},
$$

where $D F^{-k}(m): T M_{m} \rightarrow T M_{F^{-k}(m)}$ is the map induced by $F^{-k}$.

Choose a Riemannian metric for $T M$, and let | | be the corresponding norm. Define $\alpha^{*}(m)=\inf \left\{\alpha>0:\left|v^{-k}\right| / \alpha^{k} \rightarrow 0\right.$ as $k \rightarrow \infty$ for all $\left.v^{0} \in E_{m}\right\}$, $\rho^{*}(m)=\inf \left\{\rho>0:\left[\left|v^{-k}\right| /\left|w^{-k}\right|\right] / \rho^{k} \rightarrow 0\right.$ as $k \rightarrow \infty$ for all $\left.v^{0} \in E_{m}, w^{0} \in N_{m}\right\}$. The pair $(\Lambda, E)$ is called an invariant set with expanding structure if $\alpha^{*}(m)<1, \rho^{*}(m)<1$ for all $m \in \Lambda$. This definition follows the form developed in [1].

LEMmA. $\alpha^{*}$ and $\rho^{*}$ are constant on orbits and do not depend on the choice of $N$ or the metric for TM.

Local ExPANDING FAmily TheOREm. Let $(\Lambda, E)$ be an invariant set with expanding structure. Then there is a family of compact manifolds

AMS (MOS) subject classifications (1970). Primary 34D20, 34D35, 58F10. 
with boundary

$$
\overline{W_{\text {loc }}^{E}}(m)=W_{\text {loc }}^{E}(m) \cup \partial W_{\text {loc }}^{E}(m), \quad m \in \Lambda,
$$

invariant in the sense that

$$
F^{-1}\left(\overline{W_{\text {loc }}^{E}}(m)\right) \subset W_{\text {loc }}^{E}\left(F^{-1}(m)\right) .
$$

$W_{\mathrm{loc}}^{E}(m)$ is $C^{r}$ diffeomorphic to a closed ball in the fiber $E_{m}$, and is tangent to $E_{m}$ at $m . \overline{W_{\mathrm{loc}}^{E}}(m)$ depends continuously on $m$.

Suppose now that $U=V=M$, and define

$$
W^{E}(m)=\bigcup_{j=0}^{\infty} F^{j}\left(\overline{W_{\text {loc }}^{E}}\left(F^{-j}(m)\right)\right)
$$

It is easy to see that $W^{E}(m)$ is $C^{r}$ diffeomorphic to $E_{m}$, and is tangent to $E_{m}$ at $m$. The family $\left\{W^{E}(m): m \in \Lambda\right\}$ satisfies the invariance condition

$$
F^{-1}\left(W^{E}(m)\right)=W^{E}\left(F^{-1}(m)\right),
$$

and we have the following theorem.

Metric Characterization Theorem. Let $d$ denote the geodesic distance in $M$. Suppose $\alpha^{*}(m)<\alpha \leqq 1$ and $\rho^{*}(m)<\rho \leqq 1$ for all $m \in \Lambda$. Then

(i) If $p \in W^{E}(m)$, then $d\left(F^{-k}(p), F^{-k}(m)\right) / \alpha^{k} \rightarrow 0$ as $k \rightarrow \infty$.

(ii) If $p \in W^{E}(m), q \notin W^{E}(m)$, and $d\left(F^{-k}(q), F^{-k}(m)\right) \rightarrow 0$ as $k \rightarrow \infty$, then $\left[d\left(F^{-k}(p), F^{-k}(m)\right) / d\left(F^{-k}(q), F^{-k}(m)\right)\right] / \rho^{k} \rightarrow 0$ as $k \rightarrow \infty$.

2. Expanding families over invariant manifolds. Let $U$ and $V$ be open subsets of the $C^{r}$ manifold $M, 1 \leqq r \leqq \infty$, and let $F: U \rightarrow V$ be a $C^{r}$ diffeomorphism. Suppose that $\bar{\Lambda}$ is a $C^{1}$ compact manifold with boundary, properly embedded in $M$ and weakly overflowing invariant under $F$. Then also $T \bar{\Lambda}$ is weakly overflowing invariant under $T F$. Let $I \subset T M \mid \bar{\Lambda}$ be a weakly overflowing invariant subbundle containing $T \Lambda$. Choose any complement $J$ of $T \Lambda$ in $I$, and any complement $N$ of $I$ in $T M \mid \bar{\Lambda}$. Then $T M \mid \bar{\Lambda}$ splits as $T \Lambda \oplus J \oplus N$, with projections $\pi^{\Lambda}, \pi^{J}, \pi^{N}$.

For any $m \in \bar{\Lambda}$, and any $v^{0} \in T_{m} \Lambda, w^{0} \in J_{m}, x^{0} \in N_{m}$, let

$$
v^{-k}=D F^{-k}(m) v^{0}, \quad w^{-k}=\pi^{J} D F^{-k}(m) w^{0}, \quad x^{-k}=\pi^{N} D F^{-k}(m) x^{0} .
$$

Choose any metric for $T M$ and let | | be the corresponding norm. Define $\alpha_{1}^{*}(m)=\inf \left\{\alpha>0:\left|w^{-k}\right| / \alpha^{k} \rightarrow 0\right.$ as $k \rightarrow \infty$ for all $\left.w^{0} \in J_{m}\right\}$,

$\rho_{1}^{*}(m)=\inf \left\{\rho>0:\left[\left|w^{-k}\right| /\left|v^{-k}\right|\right] / \rho^{k} \rightarrow 0\right.$ as $k \rightarrow \infty$ for all $\left.v^{0} \in T_{m} \Lambda, w^{0} \in J_{m}\right\}$, $\rho_{2}^{*}(m)=\inf \left\{\rho>0:\left[\left|w^{-k}\right| /\left|x^{-k}\right|\right] / \rho^{k} \rightarrow 0\right.$ as $k \rightarrow \infty$ for all $\left.w^{0} \in J_{m}, x^{0} \in N_{m}\right\}$. 
LEMMA. $\alpha_{1}^{*}, \rho_{1}^{*}$ and $\rho_{2}^{*}$ are constant on orbits of $F$ and do not depend on the choice of a metric for TM. If $J^{\prime}$ and $N^{\prime}$ are bundles such that $I=$ $T \Lambda \oplus J^{\prime}$ and $T M \mid \bar{\Lambda}=T \Lambda \oplus J^{\prime} \oplus N^{\prime}$, then the corresponding functions are equal.

LEMma. If $\rho_{1}^{*}(m)<1$ for all $m \in \bar{\Lambda}$, there is a unique bundle $E \subset I$ such that $I=T \Lambda \oplus E$ and $E$ is weakly overflowing under $T F$.

The pair $(\bar{\Lambda}, I)$ is called an invariant manifold with expanding structure if $\alpha_{1}^{*}(m)<1, \rho_{1}^{*}(m)<1$, and $\rho_{2}^{*}(m)<1$ for all $m \in \bar{\Lambda}$.

Expanding MANIFold TheOREM. Let $(\bar{\Lambda}, I)$ be an invariant manifold with expanding structure. Let $E$ be the bundle of the previous lemma, and let $\alpha^{*}$ and $\rho^{*}$ be defined for $(\bar{\Lambda}, E)$ as in $\S 1$. Then

(i) $(\bar{\Lambda}, E)$ is an invariant set with expanding structure.

(ii) $\alpha^{*}(m)=\alpha_{1}^{*}(m)$ for all $m \in \bar{\Lambda}$.

(iii) If $\rho_{1}^{*}(m)<\rho \leqq 1$ and $\rho_{2}^{*}(m)<\rho \leqq 1$ for all $m \in \bar{\Lambda}$, then $\rho^{*}(m)<\rho \leqq 1$ for all $m \in \bar{\Lambda}$.

(iv) The fibers $W_{\mathrm{loc}}^{E}(m)$ depend continuously on $m$.

(v) $W_{\mathrm{loc}}^{E}(m) \cap W_{\mathrm{loc}}^{E}\left(m^{\prime}\right)=\varnothing$ unless $m=m^{\prime}$.

(vi) $W_{\mathrm{loc}}^{E}=\bigcup_{m \in \bar{\Lambda}} W_{\mathrm{loc}}^{E}(m)$ is a $C^{0}$ weakly overflowing invariant manifold.

(vii) If $p \in W_{\mathrm{loc}}^{E}(m), q \in W_{\mathrm{loc}}^{E}\left(m^{\prime}\right)$, then

$$
d\left(F^{-k}(p), F^{-k}(m)\right) / d\left(F^{-k}(q), F^{-k}(m)\right) \rightarrow 0 \quad \text { as } k \rightarrow \infty,
$$

unless $m=m^{\prime}$.

As in $\S 1$, there is a global construction in case $U=V=M$.

3. Asymptotic stability with asymptotic phase. Let $U$ and $V$ be open subsets of a $C^{\mathbf{1}}$ manifold $M$, and let $F: U \rightarrow V$ be a diffeomorphism. Let $\bar{\Lambda}=\Lambda \cup \partial \Lambda$ be a manifold with boundary satisfying the overflowing invariance condition $\bar{\Lambda} \subset F(\Lambda)$, and suppose $\Lambda$ is asymptotically stable under $F^{-1}$.

We say that $\Lambda$ has unique asymptotic phase if there is a neighborhood $N$ of $\Lambda$ such that for each $p \in N$, there exists in $m(p) \in \Lambda$ such that

(i) $d\left(F^{-k}(p), F^{-k}(m(p))\right) \rightarrow 0$ as $k \rightarrow \infty$;

(ii) if $m \in \Lambda, m \neq m(p)$, then

$$
d\left(F^{-k}(p), F^{-k}(m(p))\right) / d\left(F^{-k}(p), F^{-k}(m)\right) \rightarrow 0 \quad \text { as } k \rightarrow \infty .
$$

THEOREM. Let $(\bar{\Lambda}, T M \mid \bar{\Lambda})$ be an overflowing invariant manifold with expanding structure. Then the set $W_{\mathrm{loc}}^{E}$ of the Expanding Manifold Theorem is a neighborhood of $F^{-1}(\bar{\Lambda})$. Hence $\bar{\Lambda}$ is asymptotically stable with unique asymptotic phase. 
When $\Lambda$ is a compact invariant manifold satisfying the hypotheses of the perturbation theorem (Theorem 3) of [1], $\Lambda$ and its unstable bundle form an invariant manifold with expanding structure. Hence the unstable manifold has an invariant foliation into expanding fibers, and $\Lambda$ has asymptotic phase as a subset of its unstable manifold.

4. Smooth dependence on $m$. The manifold $W_{\text {loc }}^{E}$ is not even $C^{1}$, in general. As in [1], the loss of smoothness is caused by attracting limit sets in $\Lambda$. A precise theorem along these lines will appear in [2].

\section{REFERENCES}

1. N. Fenichel, Persistence and smoothness of invariant manifolds for flows, Indiana Univ. Math. J. 21 (1971/72), 193-226. MR 44 \#4313.

2. - Asymptotic stability with rate conditions, Indiana Univ. Math. J. (to appear).

Department of Mathematics, HaRvard University, CAmbridge, Massachusetts 02138 